\title{
Endoplasmic reticulum stress, cell death and tumor: Association between endoplasmic reticulum stress and the apoptosis pathway in tumors (Review)
}

\author{
XIAOJING FU ${ }^{1 *}$, JUANJUAN CUI ${ }^{2 *}$, XIANGJUN MENG ${ }^{3}$, PIYU JIANG ${ }^{1}$, \\ QIULING ZHENG ${ }^{1}$, WENWEN ZHAO ${ }^{1}$ and XUEHONG CHEN ${ }^{1}$ \\ ${ }^{1}$ School of Basic Medicine, Qingdao University; ${ }^{2}$ Qingdao Municipal Hospital, Qingdao (Group); \\ ${ }^{3}$ Qingdao Mental Health Center, Qingdao, Shandong 266071, P.R. China
}

Received June 26, 2020; Accepted October 6, 2020

DOI: $10.3892 /$ or.2021.7933

\begin{abstract}
External and internal stimuli are often involved in the pathogenesis of tumors, and the deterioration of endoplasmic reticulum (ER) function within cells is also an important etiological factor of tumorigenesis resulting in the impairment of the endoplasmic reticulum, which is termed ER stress. The ER is an organelle that serves a crucial role in the process of protein synthesis and maturation, and also acts as a reservoir of calcium to maintain intracellular $\mathrm{Ca}^{2+}$ homeostasis. ER stress has been revealed to serve a critical role in tumorigenesis. In the present review, the association between ER stress-related pathways and tumor cell apoptosis is examined. Primarily, the role of ER stress in tumor cell apoptosis is discussed, and it is stipulated that ER stress, induced by drugs both directly and indirectly, promotes tumor cell apoptosis.
\end{abstract}

\section{Contents}

1. Introduction

2. UPR: A commonly defined endoplasmic reticulum stress response

3. ER stress and the tumor cell apoptosis

4. Ferroptosis and ER stress

5. Conclusion

Correspondence to: Dr Xuehong Chen, School of Basic Medicine, Qingdao University, 308 Ningxia Road, Qingdao, Shandong 266071, P.R. China

E-mail: chen-xuehong@163.com

${ }^{*}$ Contributed equally

Key words: ER stress, apoptosis, UPR, tumor, oxidative stress, autophagy, immunogenic cell death, ferroptosis

\section{Introduction}

The endoplasmic reticulum (ER) is an organelle that receives various emergency stimuli and conducts diastolic signals. When the function of the ER is impaired due to various factors, ER stress becomes manifest, as the accumulation of misfolded and unfolded proteins within the ER chamber, as well as the disturbance of intracellular $\mathrm{Ca}^{2+}$ balance (1). Microenvironmental changes of neoplastic cells, such as those due to glucosedepletion, hypoxia or anti-tumor drugs, can induce ER stress, resulting in misfolded and unfolded protein aggregation within the cavities of the ER and intracellular imbalance of substances, which can then activate the unfolded protein response (UPR) to correct misfolded proteins and relieve stress. The subsequent elevated physiological demand for protein folding can result in the accumulation of misfolded proteins within the lumen of the ER's, which is termed ER stress (2). Activation of the universal periodic review triggers two temporarily different cellular events to mitigate protein misfolding: i) An initial response to reduce protein synthesis and boost the degradation of misfolded proteins; and ii) a second transcriptional upregulation of hundreds of target genes involved in the homeostasis of global proteins (3). Under normal physiological conditions, the protein-folding machinery in the ER is capable of secretory pathway requirements. However, whenever the accumulation of misfolded proteins within the ER exceeds a tolerable threshold, the local sensors in the ER trigger an UPR to transcriptionally and translationally optimize its protein-folding capacity. Should these corrective mechanisms prove to be insufficient, the cell will undergo apoptosis (4).

Apoptosis is closely associated with the development of neoplasms and is induced by two main pathways: The internal and external pathways. The internal (endogenous) pathway is also known as mitochondria-mediated apoptosis, which is mediated by cytokines releasing and activating caspase-9 and caspase-3 (5). The external (exogenous) pathway refers to death receptor (DR)-mediated apoptosis, which activates the FAS-associated death domain (FADD) to form the death-inducing signaling complex (DISC), resulting in the downstream activation of caspase-8, -7, -6 and $-3(5,6)$. Cancer cells proliferate because of their ability to evade programmed cell death, or apoptosis (7). 
ER stress is an important factor associated with tumor growth and invasion (8). Moreover, previous studies have provided in-depth mechanistic insights into pathways via which ER stress disrupts the homeostasis of the ER, which triggers an unresolvable UPR activation, and impairs cellular metabolism and energetics, causing uncontrolled cell death (8). With the continuation or aggravation of ER stress, cancer cells are unable to re-establish ER homeostasis via UPR, and ER stress thereby acts as a pro-apoptotic factor $(5,9,10)$. Certain studies have confirmed that ER stress can also promote the apoptosis of tumor cells (11-27). Subsequently, the present study will summarize the effects of ER stress on tumor cells in recent years, especially the role of ER stress in tumor apoptosis. ER stress activation may represent a new strategy for the synthesis and application of future chemotherapeutic drugs.

\section{UPR: A commonly defined endoplasmic reticulum stress response}

The ER is not only responsible for the synthesis and folding of up to one third of cellular proteins (12) but is also an important organelle for phospholipid synthesis and $\mathrm{Ca}^{2+}$ storage (13). Different ER subregions support intracellular homeostasis and survival pathways (14), especially the smooth ER. The smooth ER, which is responsible for lipid synthesis and metabolism, as well as calcium storage, is often modified into specific domains, including plasma-associated ER and mitochondria-associated membrane formation, autophagosomes and lipid droplets (15). Under physiological or pathological stimuli, or the action of certain drugs, the increased demand for protein folding may result in the accumulation of misfolded proteins within the ER, which triggers a UPR, expanding the folding capacity of the ER and reducing its synthesis load $(2,16)$. Three branches have been identified based on ER membrane-related ER stress sensors $(12,17)$. Each branch is defined by a class of transmembrane ER-resident signaling components, which initiate stress signaling pathways including: The protein kinase and ribonuclease, inositol requiring enzyme 1 (IRE1), transcription factor (TF), activating transcription factor 6 (ATF6), and protein kinase RNA-like ER kinase (PERK) pathways (Fig. 1A) (18). Glucose-regulated protein 78 (GRP78)/binding immunoglobulin protein (BIP) is a type of peptide-binding protein, which prevents aggregation and reduces the rate of protein folding $(1,16)$. GRP78/BIP has been demonstrated to be a direct ER stress sensor leading to UPR activation (19). Changes in the $\mathrm{Ca}^{2+}$ levels of the ER, or accumulation of either misfolded or mutant proteins, can cause GRP78 to become separated from the sensors (IRE1, PERK and ATF6), thus allowing for downstream signaling activation that regulates transcription and translation in a manner that restores homeostasis in the ER (20).

IRE1-XBP1. IRE1 is a transmembrane protein of the ER, which consists of a serine/threonine kinase domain and an endoribonuclease (RNase) domain. The mammalian genome encodes two subtypes of IRE1: IRE1 $\alpha$ and IRE1 $\beta$ (21-26). The IRE1 signaling pathway is the most conservative of the three UPR signaling pathways, and some studies have reported that the IRE1 signaling pathway may be heavily involved in tumor cell proliferation, invasion and migration (22-28). Since the expression of IRE1 $\alpha$ is ubiquitous, and the expression of IRE1 $\beta$ is limited, most mammalian UPR research focuses on the IRE1 $\alpha$ pathway (23). Studies have revealed that the IRE1 $\alpha$ signaling pathway may serve a key role in the proliferation, invasion and migration of tumors such as multiple myeloma (24) and prostate $(25,26)$, breast (27) and colorectal cancer (28). Under normal physiological conditions, IRE1 $\alpha$ expression is the same as the other two signal sensors (PERK and ATF-6), with luminal domains which bind chaperone protein BIP (29). Upon the occurrence of ER stress, IRE1 $\alpha$ is separated from BIP, after which BIP is transported to the unfolded or misfolded proteins located within the ER, and IRE1 is activated into phosphorylated IRE1 $\alpha$ (Fig. 1A) (30). Phosphorylation of IRE1 $\alpha$ activates the corresponding RNA molecule, primes the mRNA encoding $\mathrm{X}$ box-binding protein 1 (XBP1), and lyses it into splintered XBP1-S. After XBP1-S binds to the UPR element (UPRE) and the ER stress-response elements I and II (ERSE-I and ERSE-II) outside the nucleus, it is able to cross the nuclear membrane and enter the nucleus $(31,32)$. The target gene initiated can alleviate ER stress by coordinating protein folding, secretion, ER-associated protein degradation (ERAD), lipid biosynthesis and ER expansion (32).

PERK-eIF $2 \alpha$. PERK is a type I transmembrane, which oligomerizes and transautophosphorylates upon BIP dissociation or following reduced ER membrane fluidity (33). Similar to IRE1, activated PERK is phosphorylated after separating from BIP, secondary to the development of ER stress, inhibiting general protein translation via phosphorylation of the eukaryotic translation initiator factor-2 (eIF2a) at serine 51, which reduces protein overload within the ER of a stressed cell (34). The growth-arrest and DNA-damage-induced transcript 34 (GADD34) bound to the serine/threonine protein phosphatase 1 (PP1) induce the dephosphorylation of eIF $2 \alpha$, fine tuning mRNA translation to restore general protein synthesis and promote cellular recovery from stress (34). This event results in the enhanced translation of cellular apoptotic inhibitors and ATF4 (Fig. 1A) (32). Furthermore, global translational inhibition increases the selective translation of the mRNA sequence encoding the transcription factor ATF4, which directly regulates the expression of the transcription factor $\mathrm{C} / \mathrm{EBP}$-homologous protein (CHOP). These two mechanisms work synergistically to induce the transcription of multiple genes, predominantly involved in three physiological activities: Amino acid biosynthesis, amino acid transport and autophagy of the intracellular recycling system (35). Among them, ATF4 upregulates the expression of autophagy-related genes, such as MAP1LC3, Atg12 and Beclin1 (36). CHOP is a recognized transcription factor that mediates cell death by exacerbating oxidative stress, when a large number of proteins are misfolded in the ER. Previous studies have demonstrated that PERK and IRE1 signaling pathways regulate both cellular survival and apoptosis, depending on the severity and duration of the ER stress $(24-28,35,37)$.

ATF6. ATF6 is also a well-researched ER transmembrane protein that binds BIP. When ER stress is triggered, the activation of ATF6 is isolated from BIP, in a similar manner to the activation of PERK and IRE1 $(16,29)$. Nonetheless, the activated ATF6 protein is translocated from the ER to the golgi apparatus in the form of a cleaved protein domain under the 

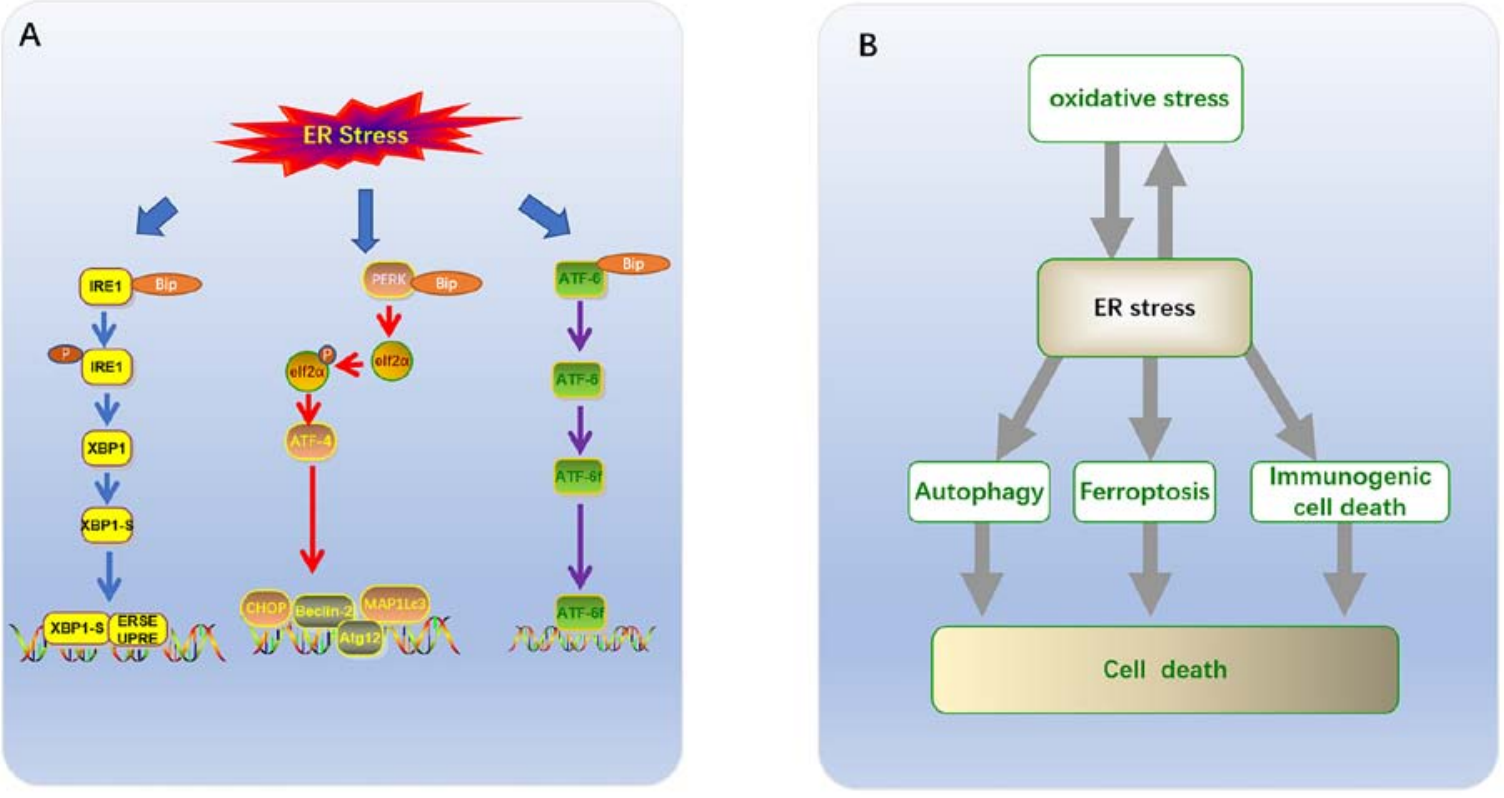

Figure 1. (A) UPR has three classical signaling pathways: i) the protein kinase and ribonuclease, IRE1; ii) the transcription factor ATF; and iii) protein kinase PERK. (B) Association of ER stress with ferroptosis, autophagy, immunogenic cell death and oxidative stress in tumor cell death. UPR, unfolded protein response; IRE1, ribonuclease inositol requiring enzyme 1; ATF6, activating transcription factor 6; PERK, protein kinase RNA-like ER kinase; UPR, the unfolded protein response.

action of regulated intramembrane proteolysis (RIP), where it is cleaved sequentially at the transmembrane site by site-1 protease (S1P) and site-2 protease (S2P) (38). This event mobilizes a $50 \mathrm{kDa}$ amino-terminal cytoplasmic fragment (ATF6f) (Fig. 1A). The ATF6f transcription factor is released into the nucleus, before binding the aforementioned ER stress response elements $(38,39)$.

ATF6 is a type II transmembrane glycoprotein which has two isoforms in mammals: ATF6a (670 amino acids) and ATF6 $\beta$ (703 amino acids); the biochemical and physiological characteristics of the former are significantly better documented than those of the latter $(39,40)$. ATF6 $\alpha$ exhibits crosstalk with XBP1s by forming heterodimers, which may drive specific gene expression programs to upregulate a subset of XBP1-dependent chaperones, oxidoreductases, as well as the quality control and degradation machinery (41). ATF6 $\alpha$ may promote the survival and adaptation of carcinoma cells by regulating a broad range of genes associated with transformation, such as ATF6 $\alpha$-Rheb-mTOR signaling $(39,42)$. In addition, ATF6 protects the $\beta$-cells of the pancreas by reducing the pressure on the ER (43). Notwithstanding, the overlapping functions of ATF6 are required to achieve transcriptional regulation of $\mathrm{CHOP}$, which is a transcription factor leading to apoptosis (40).

\section{ER stress and the tumor cell apoptosis}

Chronic ER stress and apoptosis. Several studies on a variety of cancers have confirmed that ER stress and UPR activation in a stressful microenvironment are part of a survival strategy in tumor cells (4,24-28). Nonetheless, if the adaptive response fails to restore the protein-folding function of the ER, or if severe and sustained ER stress occurs, the persistence of the UPR signal results in apoptosis, this represents an alternate signaling program known as the 'terminal UPR' $(2,4)$. PERK and IRE1 $\alpha$ are the two UPR kinases which are also involved in the occurrence of apoptosis, resulting in inevitable cell degeneration and death when ER stress cannot be resolved (Table I) (44). Under continuous and severe ER stress, IRE1 $\alpha$ induces cell apoptosis via two mechanisms: i) IRE1 $\alpha$ recruits tumor necrosis factor $\alpha(\mathrm{TNF} \alpha)$ receptor-associated factor 2 (TRAF2) and apoptosis signal regulating kinase 1 (ASK1), then activates c-Jun $\mathrm{N}$-terminal kinase (JNK) which induces apoptosis $(45,46)$; and ii) IRE1 $\alpha$ splices certain microRNAs to inhibit the expression of caspase-2, thus inducing cell apoptosis (47). Notably, activation of IRE1 $\alpha$-induced JNK is key to the induction of apoptosis in human pancreatic cells $(48,49)$.

During chronic ER stress, continuous activation of PERK results in the phosphorylation of eIF $2 \alpha$ and selective induction of ATF4, which can enhance the expression of pro-apoptotic CCAAT/enhancer binding protein (CHOP) $(32,37)$. Additionally, CHOP can induce apoptosis by downregulating the anti-apoptotic protein $\mathrm{Bcl}-2$ and upregulating the pro-apoptotic proteins (BIM and PUMA), at the transcriptional level (50). These pro-apoptotic proteins are capable of activating the transcription of a set of genes which results in activation of caspases and eventually cell death $(51,52)$. As unfolded proteins accumulate, PERK, IRE1 and GRP78 are dissolved and activated via autophosphorylation; meanwhile, ATF6 is activated by proteolytic enzymes in the golgi apparatus (53). Activated ATF6 molecules enhance the transcriptional activity of caspase- 3 by upregulating the downstream CHOP molecules (54). ATF6 $\alpha$ has been demonstrated to perform key apoptotic functions during late luteal regression in rats via the ATF6/CHOP and caspase-12 pathways (55). Moreover, in tunicamycin-exposed chondrocytes, celastrol prevented osteoarthritis by inhibiting ER-mediated apoptosis via the ATF6/CHOP signaling pathway (53). 
Table I. ER stress-related tumor apoptosis.

ER stress-related tumor apoptosis Unfolded protein response mediator(s) ER stress-related signaling pathways

(Refs.)

\begin{tabular}{llll}
\hline Chronic ER Stress & TRAF2, & PERK/ATF4/CHOP and & $\begin{array}{l}(37,53,54) \\
(37,53,54)\end{array}$ \\
& CHOP, & ATF6/CHOP & \\
caspase-12 & PERK/ATF4/CHOP & $(32,90,91)$ \\
Oxidative stress induced & ATF4 & IRE1/JNK & PERK/Elf2 $\alpha, 90,91)$ \\
Autophagy and apoptosis & JNK & IRE1 $\alpha /$ XBP1 & $(32)$ \\
& Elf2 $\alpha$ & ATF6/DAPK1 & $(32)$ \\
Ferroptosis-dependent & XBP1 & ISR & $(32)$ \\
& & PERK/eIF2 $\alpha /$ ATF4/CHOP & $(67,68)$
\end{tabular}

ER, endoplasmic reticulum; IRE1, ribonuclease inositol requiring enzyme 1; ATF6, activating transcription factor 6; PERK, protein kinase RNA-like ER kinase; TRAF2, receptor-associated factor 2; JNK, c-Jun N-terminal kinase; CHOP, C/EBP-homologous protein; XBP1, X box-binding protein 1; eIF2a, eukaryotic translation initiator factor-2; TRAF2, receptor-associated factor 2; DAPK, Death-Associated Protein kinase; ATF4, activating transcription factor-4; ISR, integrated stress response.

Furthermore, ER stress-related proteins have been shown to induce mitochondrial apoptosis in two principal ways. The exogenous or death receptor pathway is characterized by homo-oligomerization cleavage and activation of caspase-3, followed by cleavage of caspase- 8 . In the mitochondrial pathway, cytochrome $c$ s released through the mitochondrial outer membrane pores under the regulation of Bcl-2 family proteins. This activates caspase- 9 in the apoptotic corpuscle, which upregulates the expression of downstream executioner caspases, such as caspase-3 and caspase-7 $(56,57)$.

ER Stress and oxidative stress-induced apoptosis. Oxidative stress is a major driver of tumor cell proliferation, which may induce tumor survival and adaptation. The production of reactive oxygen species (ROS) can increase the mutation rate of tumor cells (58-60). The UPR regulates the level of oxidative stress by reducing global translation, while conferring cytoprotection against cellular insults via inhibition of inflammatory and apoptotic signaling pathways (32). Currently, the oxidative stress pathway is considered as a balance between cell survival and apoptosis $(59,60)$. Therefore, uncontrolled severe oxidative stress also triggers a series of pro-apoptotic signaling pathways, including ER stress and mitochondrial dysfunction, which ultimately results in cell apoptosis (60). The level of oxidative stress present in tumor cells is typically higher compared with normal cells (60). Thus, tumor cells are more likely to produce high levels of ROS, resulting in significant changes in the cytoskeleton, cell cycle arrest, DNA damage and apoptosis of tumor cells (61). Sustained production of high levels of ROS can trigger ER stress by interfering with the folding of amino acids into proteins, modifying chaperone proteins or ERAD (62). When intracellular oxidative stress levels become very high or adaptation is insufficient, apoptosis is initiated by inducing UPR-related apoptotic molecules (63).

The UPR is a commonly defined pathway in cells that regulates oxidative stress in two ways. Firstly, the UPR regulates oxidative stress by reducing the translation of transcription factors, thus inhibiting inflammatory and apoptotic signaling pathways (64). Secondly, if the stress is severe and prolonged, or if homeostasis cannot be restored, the sustained UPR will be converted to a terminal UPR, which results in cell death (65). Nude mouse tumor models were used to demonstrate that turmeric can induce the production of ROS by non-small cell lung cancer cells, activate JNK and the relevant factors of the ER stress pathway (ATF4, ATF6 and CHOP), block cell proliferation and induce cell apoptosis (Table I) (63). A study on human truncated APC colon cancer cells reported that truncated APC selective inhibitor-1 can induce ER stress-dependent JNK activation accompanied by oxidative stress, resulting in cell death (66). Besides, ROS may induce the transcription of ER oxidative reducing factor 1 (ERO1 $\alpha$ ) via the ATF4/CHOP axis, leading to cell death due to the resulting high oxidative environment in the $\operatorname{ER}(56,67)$. Overactivated ERO1 $\alpha$ can increase the production of ROS $(50,63)$. ERO1 $\alpha$ can result in inositol-1,4,5-trisphosphate receptor-mediated $\mathrm{Ca}^{2+}$ leakage in the ER, which activates $\mathrm{Ca}^{2+}$ sensing kinase CaMKII in the cytoplasm. This induces oxidative stress caused by the NADPH oxidase subunit NOX2 and leads to PER-dependent CHOP induction as a positive feedback loop in ER stress (50). Moreover, CaMKII causes activation of pro-apoptotic pathways, including Fas and mitochondrial membrane permeability transformation (50).

ER stress induced autophagy and apoptosis. Activation of the UPR is generally known to induce protective autophagy to promote cell survival during ER stress (32). PERK/Elf $2 \alpha$, IRE1 $\alpha / \mathrm{XBP} 1$, ATF6/DAPK1 and the integrated stress response (ISR) can all promote the conversion of LC3I to LC3II and form autophagosomes either directly or indirectly (Table I) $(32,68)$. In tumor cells, autophagy is widely regarded as a self-protective strategy, employed in hypoxic and nutrient-deficient microenvironments (68). In vitro and in vivo experiments confirmed that the ER stress mediated by the Brigetinib-induced autophagy response was simultaneously induced by the ER-phage as a protective mechanism to relieve excessive ER stress. Furthermore, the combination of 
Brigitinib and autophagy inhibitors was shown to significantly enhance the anti-colorectal cancer effects of Brigitinib (69). The researchers established a xenograft model of nude mice using human CRC DLD-1 cells that were subcutaneously inoculated. The study confirmed that the combinatorial treatment of Brigatinib with chloroquine resulted in a a further reduction in xenograft tumor size, growth rate and weight compared with that of Brigatinib treatment alone (69). Consequently, inhibition of autophagy (69) and acute ER stress (52) in tumor cells is currently recognized as a potential anti-tumor strategy. At present, clinical studies have proven that inhibitors of ER stress are helpful in the treatment of pancreatic cancer $(70,71)$. Nevertheless, it has been confirmed that triggering excessive ER stress induces autophagy in tumor cells, which ultimately leads to cell death (72). Accordingly, through the establishment of animal models of prostate cancer and in vitro experiments, anacardic acid was demonstrated to activate DAPK via ER stress, causing an increase in the expression of AKT and preventing its phosphorylation. This signaling pathway can upregulate the expression of LC3, Beclin-1 and Atg7 via mTOR, consequently inducing the expression of the autophagy-related protein known as Beclin-1, via suppression of mTOR phosphorylation. This results in the activation of the pro-apoptotic protein named Bax, which causes in apoptosis $(73,74)$.

ER stress-dependent immunogenic cell death in tumor cells. In response to stressors such as hypoxia and drugs, tumor cells become immunogenic, which can result in a specific cellular immune response (75). The main anticancer effector cells of the immune system are the differentiation group $\left(\mathrm{CD}^{+}\right.$ $\mathrm{T}$ cells), which can differentiate into cytotoxic $\mathrm{T}$ lymphocytes that kill tumor cells; an event known as tumor immunogenic death (ICD) $(76,77)$. ICD is associated with a series of characteristic molecular events, including the translocation of calcium netting protein (CRT) from the cytoplasm to the cell membrane surface, the release of extracellular ATP and the secretion of the high mobility group protein 1 . Immunogenic tumor cells can induce ER stress in order to trigger apoptotic cell death; moreover, it has long been hypothesized that apoptotic cell death is primarily non-immune (78). However, ER stress leads to the release of damage-associated molecular patterns (DAMPs) from the ER lumen to the plasma membrane, which can act as opsonins to facilitate the uptake of tumor associated antigens by dendritic cells (DCs) (76). During cellular stress (such as ER stress), cells can induce an immunogenic response via expression of pro-apoptotic DAMPs, including calreticulin (CALR) exposure to the cell surface, as well as the release of HMBG1 and ATP. During ER stress, Elf $2 \alpha$ is phosphorylated by the activation of PERK to induce surface exposure of CALR and heat-shock proteins (HSPs) $(79,80)$. Surface exposure to CALR is considered a critical marker of the immunogenicity of apoptosis (76). Certain specific receptors (CD91, the purinergic receptors P2Y2 and P2X7, and TLR4) recognize these DAMPs, which facilitates the phagocytosis of dying cells, attraction of DCs into the tumor bed, production of IL-1b and tumor cell antigen presentation (79). These cytokines stimulate the proliferation of $\mathrm{CD}^{+} \mathrm{T}$ cells, allowing the body to launch an anti-tumor immune response (81). Notably, the binding of DAMPs to immune cell receptors activates the UPR in immune cells resulting in an anti-tumor immune response (79).

The purpose of the present study was to explore the impact of ER-related signals in the pathogenesis and survival of tumor cells. ER stress is essential for activating intracellular signaling pathways that regulate ICD (81). Certain chemotherapeutic agents, such as anthracyclines and oxaliplatin have been demonstrated to induce CRT exposure pathways, which are activated by pre-apoptotic ER stress and phosphorylation of eIF2 kinase via PERK (Table I) (76). Subsequently, the anterograde transport of CRT from the ER to the golgi apparatus and exocytosis of CRT-containing vesicles results in the transport of CRT to the plasma membrane surface (76). Additionally, previous studies have confirmed that ROS production induces ICD via ER stress $(81,82)$. In conclusion, further investigation is imperative prior to the application of ICD induction as a therapeutic strategy against tumors.

\section{Ferroptosis and ER stress}

Ferroptosis is a type of programmed cell death distinct from apoptosis, pyroptosis and necrosis. It is caused by failure of the antioxidant defense mechanism involving glutathione (GSH/GSSG) (83). Cells that undergo ferroptosis are typically characterized by mitochondrial atrophy, increased mitochondrial membrane density and reduced intracellular NADH levels (84-86). It is generally believed that the accumulation of iron produced by lipid peroxidation and the subsequent increase in ROS levels are the key factors triggering ferroptosis (84). There is a large amount of evidence indicating that ER stress and ROS production typically interact and interfere with each other. The PERK/eIF2 $\alpha$ signaling pathway was found to regulate the production of ROS during ferroptosis $(83,87)$. After treatment with GSK414 in a mouse model of colitis, the number of necrotic cells of colonic intestinal epithelial cells, the malondialdehyde and iron contents, and the levels of ferritin light chain (FTL) and ferritin heavy chain (FTH) proteins were all reduced, suggesting that inhibition of ER stress resulted downregulation of ferroptosis, which is consistent with the findings observed from in vitro experiments (88). This study revealed that $\mathrm{HCoEpiC}$ cells, that were treated with RSL3 (a canonical inducer of ferroptosis), resulting in apparent necrotic cell death and ROS accumulation and increased levels of FTL, FTH, and PTGS2 in the RSL3-treated cells, which implied that ferroptosis had occurred (88).

Ferroptosis-dependent apoptosis. ATF4 is a basic leucine zipper transcription factor that regulates the expression levels of several UPR TARGET genes through PERK-eIF2 $\alpha$-ATF4 (89). Studies have shown that inhibition of the cystine glutamate exchange by ferroptotic agents can lead to activation of the ER stress response. Among them, the ferroptotic agent APT can lead to upregulation of ATF-4 dependent related genes, such as CHOP $(90,91)$. CHOP binds to the promoter site of the pro-apoptotic protein PUMA (p53 upregulating apoptotic regulator) during ER stress to induce the expression of PUMA $(44,90)$. Withal, this study only confirmed the correlation between ferroptosis and ER stress; reporting that ferroptotic agents induce ER stress and increase the expression of the pro-apoptotic molecular protein named PUMA through 
the ER stress-mediated PERk-eIF2 $\alpha$-ATF4-CHOP pathway, without inducing apoptosis (Table I) (44).

Ferroptosis-dependent no-apoptosis. In tumor cells, GSH intake is reduced and the production of ROS is increased, thus the sensitivity of tumor cells to ferroptosis is higher than that of normal cells due to p53 mutation and high expression of the RAS-RAF-MEK pathway $(88,92)$. ER stress has been suggested to contribute to ferroptosis via the UPR. Ferroptotic agents cause activation of an ER stress response and upregulation of the glutathione-specific $\gamma$-glutamylcyclotransferase 1 gene via inhibition of the cystine glutamate exchange. In glioma cells, ER stress has been demonstrated to attenuate docosahexaenoic acid (DHA)-induced ferroptosis by the UPR (93). DHA-induced ER stress activates the PERK/ATF4/HSPA5 pathway to resist ferroptosis and protect the cells (93). Drug resistance of tumor cells is related to the activation of ATF4, which participates in the development of chemical resistance by transcriptional regulation of membrane transporters and enzymes required for the biosynthesis of GSH in cancer cells (93). Furthermore, the activation of PERK-mediated ATF4 has a protective effect on erastin-induced ferroptosis in pancreatic cancer cells $(93,94)$. At present, studies have confirmed that ferroptosis and ER stress can be induced by ferroptotic agents (such as ART) or cystine deficiency in tumor cells due to abnormal lipid metabolism (92-94). Notwithstanding, further investigation is necessary to determine whether an upstream or downstream correlation exists between ER stress and ferroptosis.

\section{Conclusion}

Despite the fact that inhibitors targeting ER stress are being considered for clinical adjunctive therapy against tumors, reactivation of ER stress in tumor cells has become a new basis for the treatment of neoplasms, owing to deepening understanding of ER stress. ER stress with concurrent ferroptosis, ER stress leading to autophagy, as well as the synergistic effects of oxidative and ER stress can alter the homeostasis of tumor cells, which induces apoptosis (Table I). Numerous studies have published results supporting the hypothesis that ER stress promotes ferroptosis and ICD in tumor cells. Moreover, there is also evidence that ER stress is associated with other cellular death pathways caused by ICD and ferroptosis, which may represent an attractive field for the development of effective pharmacotherapies in the future.

\section{Acknowledgements}

Not applicable.

\section{Funding}

The present study was supported by the Shandong Key Research and Development Program Project (grant no. 2018GSF118124).

\section{Availability of data and materials}

Data sharing is not applicable to this article, as no datasets were generated or analyzed during the current study.

\section{Authors' contributions}

$\mathrm{XF}$ and JC wrote the manuscript, organized materials and provided concepts for the study. XM, PJ, QZ and WZ were involved in the conception of the study. XC designed the study and revised the manuscript. All authors have read and approved the final version of the manuscript.

\section{Ethics approval and consent to participate}

Not applicable.

\section{Patient consent for publication}

Not applicable.

\section{Competing interests}

The authors declare that they have no competing interests.

\section{References}

1. Sitia R and Braakman I: Quality control in the endoplasmic reticulum protein factory. Nature 426: 891-894, 2003.

2. Lu M, Lawrence DA, Marsters S, Acosta-Alvear D, Kimmig P, Mendez AS, Paton AW, Paton JC, Walter P and Ashkenazi A: Opposing unfolded-protein-response signals converge on death receptor 5 to control apoptosis. Science 345: 98-101, 2014.

3. Hetz C and Papa FR: The unfolded protein response and cell fate control. Mol Cell 69: 169-181, 2018.

4. Wang M and Kaufman RJ: The impact of the endoplasmic reticulum protein-folding environment on cancer development. Nat Rev Cancer 14: 581-597, 2014.

5. Kim C and Kim B: Anti-cancer natural products and their bioactive compounds inducing ER stress-mediated apoptosis: A review. Nutrients 10: 1021, 2018.

6. Fulda S and Debatin KM: Extrinsic versus intrinsic apoptosis pathways in anticancer chemotherapy. Oncogene 25: 4798-4811, 2006.

7. Reed JC: Dysregulation of apoptosis in cancer. J Clin Oncol 17: 2941-2953, 1999.

8. Schleicher SM, Moretti L, Varki V and Lu B: Progress in the unraveling of the endoplasmic reticulum stress/autophagy pathway and cancer: Implications for future therapeutic approaches. Drug Resist Updat 13: 79-86, 2010.

9. Ferri KF and Kroemer G: Organelle-specific initiation of cell death pathways. Nat Cell Biol 3: E255-E263, 2001.

10. Yadav RK, Chae SW, Kim HR and Chae HJ: Endoplasmic reticulum stress and cancer. J Cancer Prev 19: 75-88, 2014.

11. Park IJ, Kim MJ, Park OJ, Choe W, Kang I, Kim SS and Ha J: Cryptotanshinone induces ER stress-mediated apoptosis in HepG2 and MCF7 cells. Apoptosis 17: 248-257, 2012.

12. Pastor-Cantizano N, Ko DK, Angelos E, Pu Y and Brandizzi F: Functional diversification of ER stress responses in Arabidopsis. Trends Biochem Sci 45: 123-136, 2020.

13. Anelli T and Sitia R: Protein quality control in the early secretory pathway. EMBO J 27: 315-327, 2008.

14. Lynes EM and Simmen T: Urban planning of the endoplasmic reticulum (ER): How diverse mechanisms segregate the many functions of the ER. Biochim Biophys Acta 1813: 1893-1905, 2011.

15. English AR, Zurek N and Voeltz GK: Peripheral ER structure and function. Curr Opin Cell Biol 21: 596-602, 2009.

16. Wang $M$ and Kaufman RJ: Protein misfolding in the endoplasmic reticulum as a conduit to human disease. Nature 529: 326-335, 2016.

17. Hayashi T, Rizzuto R, Hajnoczky G and Su TP: MAM: More than just a housekeeper. Trends Cell Biol 19: 81-88, 2009.

18. Walter P and Ron D: The unfolded protein response: From stress pathway to homeostatic regulation. Science 334: 1081-1086, 2011.

19. Adams CJ, Kopp MC, Larburu N, Nowak PR and Ali MMU: Structure and molecular mechanism of ER stress signaling by the unfolded protein response signal activator IRE1. Front Mol Biosci 6: 11, 2019. 
20. Hollien $\mathbf{J}$ and Weissman JS: Decay of endoplasmic reticulum-localized mRNAs during the unfolded protein response. Science 313: 104-107, 2006.

21. Lin JH, Li H, Yasumura D, Cohen HR, Zhang C, Panning B, Shokat KM, Lavail MM and Walter P: IRE1 signaling affects cell fate during the unfolded protein response. Science 318 : 944-949, 2007.

22. Kim H, Bhattacharya A and Qi L: Endoplasmic reticulum quality control in cancer: Friend or foe. Semin Cancer Biol 33: 25-33, 2015.

23. Chen Y and Brandizzi F: IRE1: ER stress sensor and cell fate executor. Trends Cell Biol 23: 547-555, 2013.

24. Cross BC, Bond PJ, Sadowski PG, Jha BK, Zak J, Goodman JM, Silverman RH, Neubert TA, Baxendale IR, Ron D and Harding HP: The molecular basis for selective inhibition of unconventional mRNA splicing by an IRE1-binding small molecule. Proc Natl Acad Sci USA 109: E869-E878, 2012.

25. Liu J, Xiao M, Li J, Wang D, He Y, He J, Gao F, Mai L, Li Y, Liang Y, et al: Activation of UPR signaling pathway is associated with the malignant progression and poor prognosis in prostate cancer. Prostate 77: 274-281, 2017

26. Sheng X, Nenseth HZ, Qu S, Kuzu OF, Frahnow T, Simon L, Greene S, Zeng Q, Fazli L, Rennie PS, et al: IRE1 $\alpha$-XBP1s pathway promotes prostate cancer by activating c-MYC signaling. Nat Commun 10: 323, 2019.

27. Rajapaksa G, Nikolos F, Bado I, Clarke R, Gustafsson JÅ and Thomas C: ER $\beta$ decreases breast cancer cell survival by regulating the IRE1/XBP-1 pathway. Oncogene 34: 4130-4141, 2015.

28. Niederreiter L, Fritz TM, Adolph TE, Krismer AM, Offner FA, Tschurtschenthaler M, Flak MB, Hosomi S, Tomczak MF, Kaneider NC, et al: ER stress transcription factor Xbp1 suppresses intestinal tumorigenesis and directs intestinal stem cells. J Exp Med 210: 2041-2056, 2013.

29. Rutkowski DT and Kaufman RJ: A trip to the ER: Coping with stress. Trends Cell Biol 14: 20-28, 2004

30. Pincus D, Chevalier MW, Aragón T, Van Anken E, Vidal SE, El-Samad $\mathrm{H}$ and Walter P: BiP binding to the ER-stress sensor Ire1 tunes the homeostatic behavior of the unfolded protein response. PLoS Biol 8: e1000415, 2010.

31. Wang D, Hou C, Cao Y, Cheng Q, Zhang L, Li H, Feng L and Shen Y: XBP1 activation enhances MANF expression via binding to endoplasmic reticulum stress response elements within MANF promoter region in hepatitis B. Int J Biochem Cell Biol 99: 140-146, 2018.

32. Bhardwaj M, Leli NM, Koumenis C and Amaravadi RK: Regulation of autophagy by canonical and non-canonical ER stress responses. Semin Cancer Biol 66: 116-128, 2020.

33. Volmer R, Van Der Ploeg K and Ron D: Membrane lipid saturation activates endoplasmic reticulum unfolded protein response transducers through their transmembrane domains. Proc Nat Acad Sci USA 110: 4628-4633, 2013.

34. Choy MS, Yusoff P, Lee IC, Newton JC, Goh CW, Page R, Shenolikar S and Peti W: Structural and functional analysis of the GADD34:PP1 eIF2 $\alpha$ phosphatase. Cell Rep 11: 1885-1891, 2015.

35. B'chir W, Maurin AC, Carraro V, Averous J, Jousse C, Muranishi Y, Parry L, Stepien G, Fafournoux P and Bruhat A: The eIF2 $\alpha /$ ATF4 pathway is essential for stress-induced autophagy gene expression. Nucleic Acids Res 41: 7683-7699, 2013.

36. Luhr M, Torgersen ML, Szalai P, Hashim A, Brech A, Staerk J and Engedal N: The kinase PERK and the transcription factor ATF4 play distinct and essential roles in autophagy resulting from tunicamycin-induced ER stress. J Biol Chem 294: 8197-8217, 2019.

37. Rozpedek W, Pytel D, Mucha B, Leszczynska H, Diehl JA and Majsterek I: The role of the PERK/eIF2 $\alpha / \mathrm{ATF} 4 / \mathrm{CHOP}$ signaling pathway in tumor progression during endoplasmic reticulum stress. Curr Mol Med 16: 533-544, 2016.

38. Shen J, Chen X, Hendershot L and Prywes R: ER stress regulation of ATF6 localization by dissociation of BiP/GRP78 binding and unmasking of Golgi localization signals. Dev Cell 3: 99-111, 2002.

39. Hillary RF and Fitzgerald U: A lifetime of stress: ATF6 in development and homeostasis. J Biomed Sci 25: 48, 2018.

40. Correll RN, Grimes KM, Prasad V, Lynch JM, Khalil H and Molkentin JD: Overlapping and differential functions of ATF6 $\alpha$ versus ATF6 $\beta$ in the mouse heart. Sci Rep 9: 2059, 2019.

41. Shoulders MD, Ryno LM, Genereux JC, Moresco JJ, Tu PG, Wu C, Yates JR III, Su AI, Kelly JW and Wiseman RL: Stress-independent activation of XBP1s and/or ATF6 reveals three functionally diverse ER proteostasis environments. Cell Rep 3: 1279-1292, 2013.
42. Schewe DM and Aguirre-Ghiso JA: ATF6alpha-Rheb-mTOR signaling promotes survival of dormant tumor cells in vivo. Proc Natl Acad Sci USA 105: 10519-10524, 2008.

43. Usui M, Yamaguchi S, Tanji Y, Tominaga R, Ishigaki Y, Fukumoto M, Katagiri H, Mori K, Oka Y and Ishihara $\mathrm{H}$ : Atf6 $\alpha$-null mice are glucose intolerant due to pancreatic $\beta$-cell failure on a high-fat diet but partially resistant to diet-induced insulin resistance. Metabolism 61: 1118-1128, 2012.

44. Urra H, Dufey E, Lisbona F, Rojas-Rivera D and Hetz C: When ER stress reaches a dead end. Biochim Biophys Acta 1833: 3507-3517, 2013

45. Tabas I and Ron D: Integrating the mechanisms of apoptosis induced by endoplasmic reticulum stress. Nat Cell Biol 13: 184-190, 2011.

46. Chen L, Xu S, Liu L, Wen X, Xu Y, Chen J and Teng J: Cab45S inhibits the ER stress-induced IRE1-JNK pathway and apoptosis via GRP78/BiP. Cell Death Dis 5: e1219, 2014.

47. Upton JP, Wang L, Han D, Wang ES, Huskey NE, Lim L, Truitt M, Mcmanus MT, Ruggero D, Goga A, et al: IRE1 $\alpha$ cleaves select microRNAs during ER stress to derepress translation of proapoptotic Caspase-2. Science 338: 818-822, 2012.

48. Brozzi F, Nardelli TR, Lopes M, Millard I, Barthson J, Igoillo-Esteve M, Grieco FA, Villate O, Oliveira JM, Casimir M, et al: Cytokines induce endoplasmic reticulum stress in human, rat and mouse beta cells via different mechanisms. Diabetologia 58: 2307-2316, 2015.

49. Brozzi F, Gerlo S, Grieco FA, Juusola M, Balhuizen A, Lievens S, Gysemans C, Bugliani M, Mathieu C, Marchetti P, et al: Ubiquitin $D$ regulates IRE1 $\alpha / J N K-d e p e n d e n t$ apoptosis in pancreatic beta cells. J Biol Chem 291: 12040-12056, 2016.

50. Zhang Z, Zhang L, Zhou L, Lei Y, Zhang Y and Huang C: Redox signaling and unfolded protein response coordinate cell fate decisions under ER stress. Redox Biol 25: 101047, 2019.

51. Ma Y and Hendershot LM: Delineation of a negative feedback regulatory loop that controls protein translation during endoplasmic reticulum stress. J Biol Chem 278: 34864-34873, 2003.

52. Jaud M, Philippe C, Di Bella D, Tang W, Pyronnet S, Laurell H, Mazzolini L, Rouault-Pierre K and Touriol C: Translational regulations in response to endoplasmic reticulum stress in cancers. Cells 9: 540, 2020.

53. Liu DD, Zhang BL, Yang JB and Zhou K: Celastrol ameliorates endoplasmic stress-mediated apoptosis of osteoarthritis via regulating ATF-6/CHOP signalling pathway. J Pharm Pharmacol 72: 826-835, 2020

54. Tang YH, Yue ZS, Zheng WJ, Shen HF, Zeng LR, Hu ZQ and Xiong ZF: 4-Phenylbutyric acid presents therapeutic effect on osteoarthritis via inhibiting cell apoptosis and inflammatory response induced by endoplasmic reticulum stress. Biotechnol Appl Biochem 65: 540-546, 2018.

55. Yang Y, Sun M, Shan Y, Zheng X, Ma H, Ma W, Wang Z, Pei X and Wang Y: Endoplasmic reticulum stress-mediated apoptotic pathway is involved in corpus luteum regression in rats. Reprod Sci 22: 572-584, 2015

56. Iurlaro R and Muñoz-Pinedo C: Cell death induced by endoplasmic reticulum stress. FEBS J 283: 2640-2652, 2016.

57. Masud A, Mohapatra A, Lakhani SA, Ferrandino A, Hakem R and Flavell RA: Endoplasmic reticulum stress-induced death of mouse embryonic fibroblasts requires the intrinsic pathway of apoptosis. J Biol Chem 282: 14132-14139, 2007.

58. Clarke HJ, Chambers JE, Liniker E and Marciniak SJ: Endoplasmic reticulum stress in malignancy. Cancer Cell 25: 563-573, 2014

59. Cairns RA, Harris IS and Mak TW: Regulation of cancer cell metabolism. Nat Rev Cancer 11: 85-95, 2011.

60. Zhang X, Chen M, Zou P, Kanchana K, Weng Q, Chen W, Zhong P, Ji J, Zhou H, He L and Liang G: Curcumin analog WZ35 induced cell death via ROS-dependent ER stress and $\mathrm{G} 2 / \mathrm{M}$ cell cycle arrest in human prostate cancer cells. BMC Cancer 15: 866, 2015.

61. Kong N, Ji X, Wang J, Sun X, Chen G, Fan T, Liang W, Zhang H, Xie A, Farokhzad OC and Tao W: ROS-Mediated selective killing effect of black phosphorus: Mechanistic understanding and its guidance for safe biomedical applications. Nano Lett 20: 3943-3955, 2020

62. Geraghty P, Wallace A and D'armiento JM: Induction of the unfolded protein response by cigarette smoke is primarily an activating transcription factor $4-\mathrm{C} / \mathrm{EBP}$ homologous protein mediated process. Int J Chron Obstruct Pulmon Dis 6: 309-319, 2011. 
63. Ma J, Liu J, Lu C and Cai D: Pachymic acid induces apoptosis via activating ROS-dependent JNK and ER stress pathways in lung cancer cells. Cancer Cell Int 15: 78, 2015.

64. Cubillos-Ruiz JR, Bettigole SE and Glimcher LH: Tumorigenic and immunosuppressive effects of endoplasmic reticulum stress in cancer. Cell 168: 692-706, 2017.

65. Bravo R, Parra V, Gatica D, Rodriguez AE, Torrealba N, Paredes F, Wang ZV, Zorzano A, Hill JA, Jaimovich E, et al: Endoplasmic reticulum and the unfolded protein response: Dynamics and metabolic integration. Int Rev Cell Mol Biol 301: 215-290, 2013

66. Zhang L, Kim SB, Luitel K and Shay JW: Cholesterol depletion by TASIN-1 induces apoptotic cell death through the ER stress/ROS/JNK signaling in colon cancer cells. Mol Cancer Ther 17: 943-951, 2018.

67. Marciniak SJ, Yun CY, Oyadomari S, Novoa I, Zhang Y, Jungreis R, Nagata K, Harding HP and Ron D: CHOP induces death by promoting protein synthesis and oxidation in the stressed endoplasmic reticulum. Genes Dev 18: 3066-3077, 2004

68. Rouschop KM, Van den Beucken T, Dubois L, Niessen H, Bussink J, Savelkouls K, Keulers T, Mujcic H, Landuyt W, Voncken JW, et al: The unfolded protein response protects human tumor cells during hypoxia through regulation of the autophagy genes MAP1LC3B and ATG5. J Clin Invest 120: 127-141, 2010.

69. Zhang Z, Gao W, Zhou L, Chen Y, Qin S, Zhang L, Liu J, He Y, Lei Y, Chen HN, et al: Repurposing brigatinib for the treatment of colorectal cancer based on inhibition of ER-phagy. Theranostics 9: 4878-4892, 2019.

70. Wang J, Qi Q, Zhou W, Feng Z, Huang B, Chen A, Zhang D, $\mathrm{Li} W$, Zhang Q, Jiang Z, et al: Inhibition of glioma growth by flavokawain $B$ is mediated through endoplasmic reticulum stress induced autophagy. Autophagy 14: 2007-2022, 2018.

71. Atkins C, Liu Q, Minthorn E, Zhang SY, Figueroa DJ, Moss K, Stanley TB, Sanders B, Goetz A, Gaul N, et al: Characterization of a novel PERK kinase inhibitor with antitumor and antiangiogenic activity. Cancer Res 73: 1993-2002, 2013.

72. Rah B, Ur Rasool R, Nayak D, Yousuf SK, Mukherjee D Kumar LD and Goswami A: PAWR-mediated suppression of BCL2 promotes switching of 3-azido withaferin A (3-AWA)-induced autophagy to apoptosis in prostate cancer cells. Autophagy 11: 314-331, 2015.

73. Fujiwara N, Usui T, Ohama T and Sato K: Regulation of beclin 1 protein phosphorylation and autophagy by protein phosphatase $2 \mathrm{~A}$ (PP2A) and death-associated protein kinase 3 (DAPK3). J Biol Chem 291: 10858-10866, 2016.

74. Tan J, Jiang X, Yin G, He L, Liu J, Long Z, Jiang Z and Yao K Anacardic acid induces cell apoptosis of prostatic cancer through autophagy by ER stress/DAPK3/Akt signaling pathway. Oncol Rep 38: 1373-1382, 2017.

75. Wang YJ, Fletcher R, Yu J and Zhang L: Immunogenic effects of chemotherapy-induced tumor cell death. Genes Dis 5: 194-203, 2018.

76. Zitvogel L, Kepp O, Senovilla L, Menger L, Chaput N and Kroemer G: Immunogenic tumor cell death for optimal anticancer therapy: The calreticulin exposure pathway. Clin Cancer Res 16: 3100-3104, 2010.

77. Radogna F and Diederich M: Stress-induced cellular responses in immunogenic cell death: Implications for cancer immunotherapy. Biochem Pharmacol 153: 12-23, 2018.

78. Obeid M, Tesniere A, Ghiringhelli F, Fimia GM, Apetoh L, Perfettini JL, Castedo M, Mignot G, Panaretak is T, Casares N, et al: Calreticulin exposure dictates the immunogenicity of cancer cell death. Nat Med 13: 54-61, 2007.
79. Obacz J, Avril T, Rubio-Patiño C, Bossowski JP, Igbaria A, Ricci JE and Chevet E: Regulation of tumor-stroma interactions by the unfolded protein response. FEBS J 286: 279-296, 2019.

80. Panaretakis T, Kepp O, Brockmeier U, Tesniere A, Bjorklund AC, Chapman DC, Durchschlag M, Joza N, Pierron G, Van Endert P, et al: Mechanisms of pre-apoptotic calreticulin exposure in immunogenic cell death. EMBO J 28: 578-590, 2009.

81. Li W, Yang J, Luo L, Jiang M, Qin B, Yin H, Zhu C, Yuan X, Zhang J, Luo Z, et al: Targeting photodynamic and photothermal therapy to the endoplasmic reticulum enhances immunogenic cancer cell death. Nat Commun 10: 3349, 2019.

82. Deng H, Zhou Z, Yang W, Lin LS, Wang S, Niu G, Song J and Chen X: Endoplasmic reticulum targeting to amplify immunogenic cell death for cancer immunotherapy. Nano Lett 20: 1928-1933, 2020.

83. Lee YS, Lee DH, Choudry HA, Bartlett DL and Lee YJ: Ferroptosis-induced endoplasmic reticulum stress: Cross-talk between ferroptosis and apoptosis. Mol Cancer Res 16: 1073-1076, 2018

84. Dixon SJ, Lemberg KM, Lamprecht MR, Skouta R, Zaitsev EM, Gleason CE, Patel DN, Bauer AJ, Cantley AM, Yang WS, et al: Ferroptosis: An iron-dependent form of nonapoptotic cell death. Cell 149: 1060-1072, 2012.

85. Hassannia B, Vandenabeele P and Vanden Berghe T: Targeting ferroptosis to iron out cancer. Cancer Cell 35: 830-849, 2019.

86. Hartman ML: Non-apoptotic cell death signaling pathways in melanoma. Int J Mol Sci 21: 2980, 2020.

87. Park EJ, Park YJ, Lee SJ, Lee K and Yoon C: Whole cigarette smoke condensates induce ferroptosis in human bronchial epithelial cells. Toxicol Lett 303: 55-66, 2019.

88. Xu M, Tao J, Yang Y, Tan S, Liu H, Jiang J, Zheng F and Wu B: Ferroptosis involves in intestinal epithelial cell death in ulcerative colitis. Cell Death Dis 11: 86, 2020.

89. Hong SH, Lee DH, Lee YS, Jo MJ, Jeong YA, Kwon WT, Choudry HA, Bartlett DL and Lee YJ: Molecular crosstalk between ferroptosis and apoptosis: Emerging role of ER stress-induced p53-independent PUMA expression. Oncotarget 8: 115164-115178, 2017.

90. Su N and Kilberg MS: C/EBP homology protein (CHOP) interacts with activating transcription factor 4 (ATF4) and negatively regulates the stress-dependent induction of the asparagine synthetase gene. J Biol Chem 283: 35106-35117, 2008.

91. Ghosh AP, Klocke BJ, Ballestas ME and Roth KA: CHOP potentially co-operates with FOXO3a in neuronal cells to regulate PUMA and BIM expression in response to ER stress. PLoS One 7: e39586, 2012.

92. Zhou B, Liu J, Kang R, Klionsky DJ, Kroemer G and Tang D: Ferroptosis is a type of autophagy-dependent cell death. Semin Cancer Biol 66: 89-100, 2020.

93. Chen Y, Mi Y, Zhang X, Ma Q, Song Y, Zhang L, Wang D, Xing J, Hou B, Li H, et al: Dihydroartemisinin-induced unfolded protein response feedback attenuates ferroptosis via PERK/ATF4/HSPA5 pathway in glioma cells. J Exp Clin Cancer Res 38: 402, 2019.

94. Dixon SJ, Patel DN, Welsch M, Skouta R, Lee ED, Hayano M, Thomas AG, Gleason CE, Tatonetti NP, Slusher BS and Stockwell BR: Pharmacological inhibition of Cystine-glutamate exchange induces endoplasmic reticulum stress and ferroptosis. Elife 3: e02523, 2014.

This work is licensed under a Creative Commons Attribution-NonCommercial-NoDerivatives 4.0 International (CC BY-NC-ND 4.0) License. 